\title{
高导热膨胀石墨/硬脂酸定形相变储能复合材料的 制备及储放热特性
}

\author{
翟天尧, 李廷贤*，仵斯，王如竹 \\ 上海交通大学制冷与低温工程研究所, 上海 200240 \\ * 联系人, E-mail: Litx@sjtu.edu.cn
}

2017-07-24 收稿, 2017-09-04 修回, 2017-09-05 接受, 2017-12-07 网络版发表 国家优秀青年科学基金(51522604)和国家创新研究群体科学基金(51521004)资助

\begin{abstract}
摘要采用“熔融吸附-模压成型”的方法制备了膨胀石墨(EG)为多孔基体、硬脂酸(SA)为相变材料的EG/SA定形 相变储能复合材料, 通过微观形貌、热物性和热稳定性测试, 分析了膨胀石墨的膨胀度对EG/SA定形相变储能复 合材料的热性能影响规律, 并通过加热/冷却实验对不同参数的定形相变储能复合材料的储/放热性能进行了分析 比较. 研究表明采用高膨胀度EG更有利于提高 SA在EG多孔基体中的分布均匀性和储能复合材料的热导率, 当 EG质量分数为 $20 \%$ 时其径向导热系数最高达 $19.6 \mathrm{~W} \mathrm{~m}^{-1} \mathrm{~K}^{-1}$, 相比纯 $\mathrm{SA}$ 提高了 110 倍; EG的高膨胀度对 SA相变过 程中的液相封装具有明显的改善作用, 高膨胀度EG为基体的定形相变储能复合材料具有很好的热稳定性; EG/SA 定形相变储能复合材料的储/放热时间约为纯相变材料的 $1 / 8 \sim 1 / 4$ ，具有高导热、无泄漏等优点.
\end{abstract}

关键词＼cjkstart膨胀石墨, 硬脂酸, 相变储能, 定形复合材料, 导热系数, 热性能

储能技术可以克服能量供需在时间和空间上的 不匹配矛盾, 是提高能源利用效率和可靠性的有效 手段, 尤其在可再生能源的合理分配中扮演着重要 的角色. 一般来说, 储热技术主要分为显热储能、潜 热储能和热化学储能, 相比显热储能, 相变潜热储能 具有比较高的能量密度和较稳定的蓄放热温度 ${ }^{[1]}$, 因 其优良的储热性能和显著的节能效果广泛应用于制 冷系统 ${ }^{[2]}$ 、建筑空调 ${ }^{[3]}$ 、太阳热能利用 ${ }^{[4]}$ 、电子元件 冷却 ${ }^{[5]}$ 等领域, 已成为能源利用领域的研究热点.

相变材料可分为有机物质和无机物质两种, 由 于有机物具有相变温度适应性好、相变潜热大、理化 性能稳定等优点, 因而在太阳能、余热利用等方面受 到广泛关注 ${ }^{[6]}$. 近年来, 将有机相变材料与高导热无 机基质复合制备结构稳定的复合相变材料得到科研 工作者的广泛研究, 膨胀石墨基复合相变材料则是
其中最具代表性的一类复合相变材料. 膨胀石墨作 为复合相变材料中的吸附基质，一方面可以增强传 热性能, 降低材料内部的温差, 大大减少储/放热的 时间 ${ }^{[7]}$; 另一方面可以对相变材料进行定形封装, 克 服了固-液相变材料在相变时的流动性问题 ${ }^{[8]}$, 大大 推动了相变储能材料的应用发展.

高学农等人 ${ }^{[9]}$ 采用摚拌吸附法制备了石蜡质量 分数为 $90 \%$ 的石蜡/膨胀石墨相变储能复合材料, 测 得其导热系数为 $1.738 \mathrm{~W} \mathrm{~m}^{-1} \mathrm{~K}^{-1}$, 相比纯石蜡 $(0.3608$ $\mathrm{W} \mathrm{m}{ }^{-1} \mathrm{~K}^{-1}$ ) 提高了约 4 倍; 胡芃等人 ${ }^{[10]}$ 采用共混熔融 法制备了一系列棕㭣酸/膨胀石墨相变储热复合材料, 蓄放热速率测试显示相较于纯棕㭣酸复合相变材料 完成充放热过程所需时间显著缩短; 周卫兵等人 ${ }^{[11]}$ 通过真空熔融吸附法制备了 $\mathrm{EG}$ 质量分数为 $8 \%$ 的 $\mathrm{EG} / \mathrm{SA}$ 相变储热复合材料, 导热测试结果显示复合

引用格式：翟天尧，李廷贤，仵斯，等. 高导热膨胀石墨/硬脂酸定形相变储能复合材料的制备及储/放热特性. 科学通报, 2018, 63: 674-683 Zhai T Y, Li T X, Wu S, et al. Preparation and thermal performance of form-stable expanded graphite/stearic acid composite phase change materials with high thermal conductivity (in Chinese). Chin Sci Bull, 2018, 63: 674-683, doi: 10.1360/N972017-00831 
相变材料热导率由纯 $\mathrm{SA}$ 的 $0.18 \mathrm{~W} \mathrm{~m}^{-1} \mathrm{~K}^{-1}$ 提高到 2.52 $\mathrm{W} \mathrm{m} \mathrm{m}^{-1} \mathrm{~K}^{-1}$.

上海交通大学储能课题组 ${ }^{[12]}$ 对已有定形相变储 能复合材料的制备方法进行了改进, 采用“熔融吸附模压成型”的方法, 制备了一系列EG/SA样品, 导热 测试结果显示 $E G$ 的加人显著提高了样品的轴向和 径向热导率, 径向热导率最高达 $23.77 \mathrm{~W} \mathrm{~m}^{-1} \mathrm{~K}^{-1}$, 相 比纯硬脂酸 $\left(0.18 \mathrm{~W} \mathrm{~m}^{-1} \mathrm{~K}^{-1}\right)$ 提高了约 132 倍. 孙凯等 人 ${ }^{[13]}$ 测定了不同温度下制得的膨胀石墨对石蜡的稳 定吸附倍率和饱和吸附倍率, 发现EG对石蜡的吸附 倍率随膨化温度升高而增加, $900{ }^{\circ} \mathrm{C}$ 时饱和吸附量和 稳定吸附倍率达到最大. 王海宁等人 ${ }^{[14]}$ 利用压录法 和图像分析法研究了膨胀石墨的孔结构, 发现膨胀 石墨制备参数对膨胀石墨的孔结构有显著影响, 其 中插层电量越大、膨化温度越高, 孔径尺寸越大, 孔 径分布也越宽, 且当膨化温度高于 $800^{\circ} \mathrm{C}$ 时, 孔结构 参数趋于稳定.

目前针对膨胀石墨膨胀度对于相变储能复合材 料导热和热性能影响的研究较少, 前期本课题组研 究发现膨胀石墨基相变储能复合材料在EG含量较少 时其径向导热有时会随样品成型密度的增大反而出 现下降现象, 同时存在SA泄漏的问题, 初步分析表 明该现象与膨胀石墨的膨胀度息息相关. 本文立足 于 “熔融吸附-模压成型”的定形相变储能复合材料制 备方法 ${ }^{[12]}$, 以 $\mathrm{SA}$ 为相变材料, 两种不同膨胀度的 $\mathrm{EG}$ 为基体, 各制备了 10 种不同参数(密度为 750,800 , $850,900,950 \mathrm{~kg} \mathrm{~m}^{-3}$; EG质量分数为 $15 \%, 20 \%$, 下 同)的 EG/SA定形相变储能复合材料样品, 探讨了膨 胀石墨膨胀度对复合材料导热性能和热稳定性的影 响; 同时制备了 5 种不同参数的储/放热单元装置, 并 对其储/放热速率进行了测试和分析.

\section{1 实验}

\section{1 样品制备}

采用分析纯级硬脂酸(国药集团化学试剂有限公 司)和两种不同膨胀度的膨胀石墨为原材料制备定形 相变储能复合材料. 其中膨胀石墨 $\mathrm{a}$ (以下样品名称后 缀带 $\mathrm{a}$ 为此膨胀石 墨制备) 是将粒度为 50 目的可膨胀 石墨粉 (上海一帆石墨有限公司)在 $120^{\circ} \mathrm{C}$ 环境下干燥 $20 \mathrm{~h}$ 后置于 $600^{\circ} \mathrm{C}$ 电炉中热处理得到; 膨胀石墨 $\mathrm{b}$ (以下 样品名称后缀带 $\mathrm{b}$ 为此膨胀石墨制备)是将粒度 80 目的
可膨胀石墨(上海虎迈复合材料有限公司)在温度950 $1000^{\circ} \mathrm{C}$ 工况下热处理使其膨胀 300 倍得到. 图 1 为相同 质量工况下两种不同膨胀度的膨胀石墨的体积对比, 可以看出膨胀石墨 $\mathrm{a}$ 体积约为 $30 \mathrm{~mL} \mathrm{~g}^{-1}$, 膨胀石墨 $\mathrm{b}$ 体 积约为 $60 \mathrm{~mL} \mathrm{~g}^{-1}$, 后者膨胀程度明显高于前者.

首先使用粉碎机将颗粒状硬脂酸 SA粉碎成较细 粉末, 以便于更均匀地吸附在膨胀石墨EG孔隙中, 分别取两种不同膨胀度的膨胀石墨EG基体和粉末状 $\mathrm{SA}$ 相变材料按一定质量比(EG质量分数分别为 $15 \%$ 和 $20 \%$ )搅拌混合, 使 SA与EG尽可能均匀混合后将复 合材料置于 $80^{\circ} \mathrm{C}$ 的电炉中加热至 SA完全熔化, 利用 膨胀石墨微孔结构的毛细力吸附液态的相变材料 SA 使其填充到孔隙中. 然后取一定质量的复合材料倒 人模具中, 使用液压设备压缩得到尺寸为 $31 \mathrm{~mm} \times 31$ $\mathrm{mm} \times 15 \mathrm{~mm}$ 的定形相变储能复合材料方块, 如图2(a) 所示, 通过改变复合材料的 EG质量分数和控制样品 的成型密度 $\left(750,800,850,900,950 \mathrm{~kg} \mathrm{~m}^{-3}\right)$, 共制备 了 $\mathrm{a}, \mathrm{b}$ 两组各 10 种不同参数的定形复合材料样品, 然 后将这些样品分别切割打磨成数个 $10 \mathrm{~mm} \times 10 \mathrm{~mm} \times 3$ $\mathrm{mm}$ 径向薄片和 1 个尺寸约为 $25 \mathrm{~mm} \times 25 \mathrm{~mm} \times 14 \mathrm{~mm}$ 的 方块用于导热性能测试和循环热稳定性测试.

为了研究 $E G$ 质量分数以及压制成型处理对定形 相变储能复合材料的实际换热影响, 采用上述方法 把 $\mathrm{EG} / \mathrm{SA}$ 复合材料样品 (EG质量含量分别为 $15 \%$ 和 $20 \%$ 直接压制进尺寸为 $100 \mathrm{~mm} \times 100 \mathrm{~mm} \times 200 \mathrm{~mm}$ 的 不锈钢容器中使相变储能复合材料与容器壁面紧密 接触减少热阻, 其中定形相变储能复合材料方块尺 寸为 $100 \mathrm{~mm} \times 100 \mathrm{~mm} \times 50 \mathrm{~mm}$, 密度分别为 750 和 900 $\mathrm{kg} \mathrm{m}^{-3}$, 包含参照样品纯 $\mathrm{SA}$ (密度 $847 \mathrm{~kg} \mathrm{~m}^{-3}$ ) 在内共 制备了 5 个储/放热单元装置, 材料中心点布置PT100

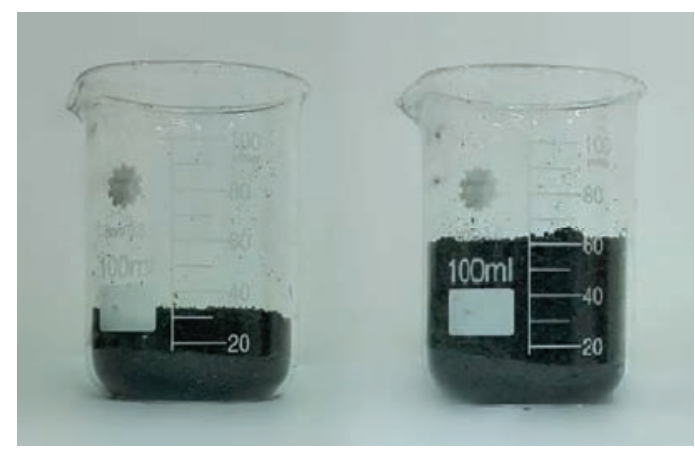

图 1 (网络版彩色)膨胀石墨 $\mathrm{a}$ (左)和膨胀石墨 $\mathrm{b}$ (右)体积对比(质量 $1 \mathrm{~g}$ ) Figure 1 (Color online) The comparison of loose volume of expanded graphite a (left) and expanded graphite b (right) per gram 


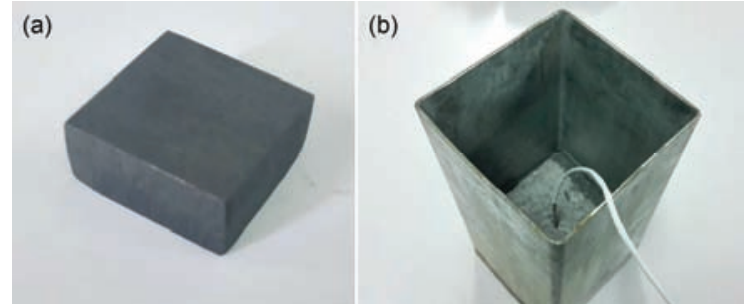

图 2 (网络版彩色) EG/SA定形相变储能复合材料样品和单元装置. (a) 复合材料方块; (b) 储能单元

Figure 2 (Color online) Form-stable EG/SA composite samples and energy storage units. (a) Composite block; (b) energy storage unit

热电阻测温, 如图2(b)所示.

\section{2 样品表征}

采用扫描电子显微镜(SEM, 美国FEI公司, Sirion 200)观察EG及EG/SA复合材料的微观形貌; 采用差 式扫描热量仪(DSC8000, 美国 Perkin-Elmer公司)测 试样品的相变温度和潜热, 升降温速率为 $5^{\circ} \mathrm{C} \mathrm{min}^{-1}$; 使用激光导热系数测量仪 (LAF447, 德国 NETZSCH 公司)测量样品导热系数, 每个样品多次测量取平均 值; 使用电烘箱对样品的热稳定性进行测试, 将样品 放在不锈钢盘中后放人 $80^{\circ} \mathrm{C}$ 电烘箱中加热, $\mathrm{SA}$ 完全 熔化后取出自然冷却至常温, 即完成 1 次储/放热循
环. 观察样品形态，如果有SA泄漏刮去样品表面析 出的SA. 每次循环后称重并记录样品重量变化, 重 复储/放热过程至所有样品均不出现明显质量和形态 变化. 进行储/放热实验时先将 5 个储/放热单元置于 $85^{\circ} \mathrm{C}$ 热水浴中完成储热过程, 然后置于 $30^{\circ} \mathrm{C}$ 冷水浴 中完成放热过程, 实验中控制热/冷源到测点的传热 距离和传热温差, 记录各单元材料中心点温度变化.

\section{2 结果与分析}

\subsection{SEM分析}

图3所示为两种不同膨胀度 $\mathrm{EG}$ 以及 $\mathrm{EG} / \mathrm{SA}$ 复合 材料的微观结构. 图3(a)和(b)所示为放大 10000倍时 两种EG的网状孔隙结构, 可以看到膨胀石墨膨胀程 度增大使得其微孔排布更加规则, 孔径和孔体积也 有所增大, 更利于SA颗粒的进人和均匀吸附.

图 3(c)和 (d) 则为放大 10000 倍时观察到的两种 EG配置的EG含量 $15 \%$ 、密度 $900 \mathrm{~kg} \mathrm{~m}^{-3}$ 的定形相变储 能复合材料样品断层表面的微观形貌, 可以看到图 3(c)中石墨片层表面SA分布不是很均匀, 部分区域 存在SA较多、晶粒较厚的现象; 而图3(d)中 SA晶粒则 薄而均匀地附着在石墨片层上, 不存在 $\mathrm{SA}$ 的堆积
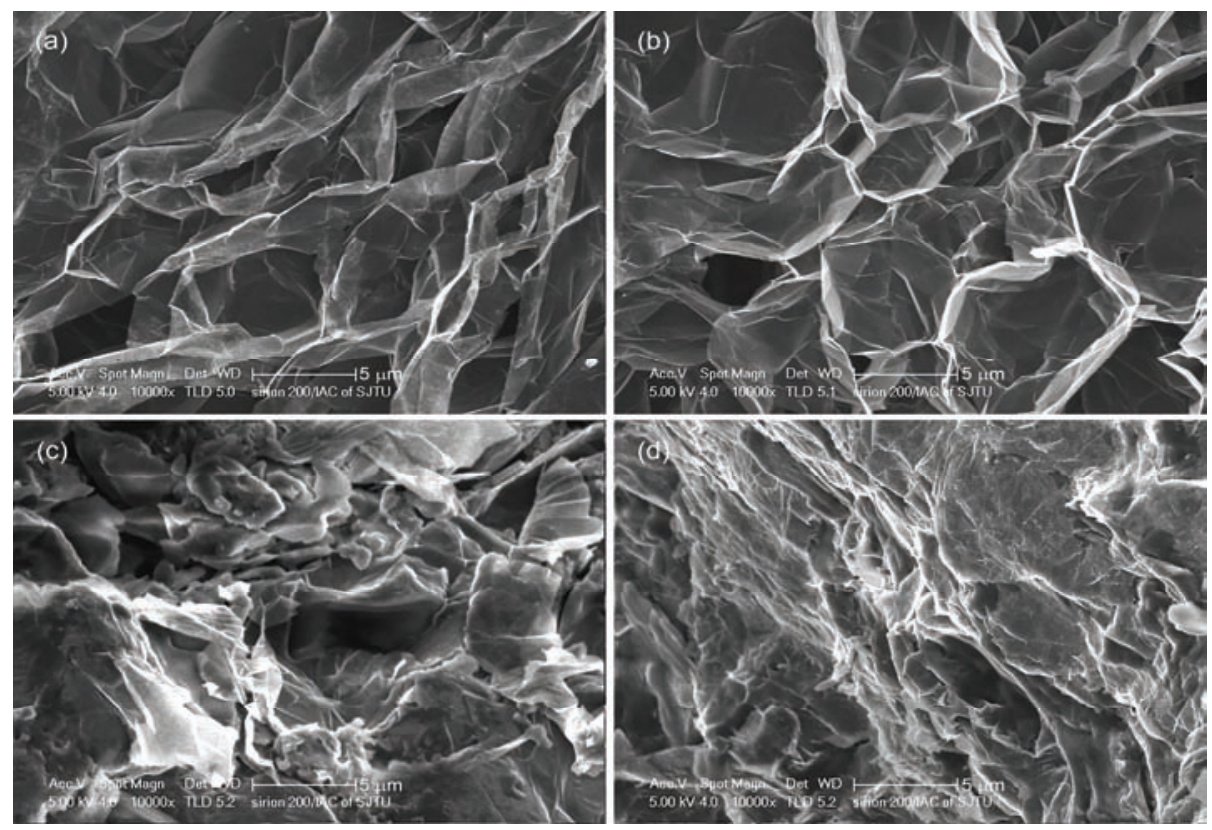

图 3 膨胀石墨及EG/SA定形相变储能复合材料SEM微观结构. (a) EG a; (b) EG b; (c) 15 wt.\%EGa/SA定形复合材料 $900 \mathrm{~kg} \mathrm{~m}{ }^{-3}$; (d) 15 wt.\% EGb/SA定形复合材料 $900 \mathrm{~kg} \mathrm{~m}^{-3}$

Figure 3 The SEM microstructures of expanded graphite matrix and form-stable EG/SA composite samples. (a) EG a; (b) EG b; (c) 15 wt.\%EGa/SA sample with $900 \mathrm{~kg} \mathrm{~m}^{-3}$; (d) $15 \mathrm{wt} \% \mathrm{EGb} / \mathrm{SA}$ sample with $900 \mathrm{~kg} \mathrm{~m}^{-3}$ 
现象. 这是因为膨胀石墨 $\mathrm{b}$ 因为有更多的微孔和更大 的孔体积, 在与 SA混合熔融吸附时 SA分布更为均 匀; 而膨胀石墨 $\mathrm{a}$ 因为膨胀度较低, 微孔结构的空间 不足以容纳所有 SA, 部分SA没有进人微孔当中, 而 是附着在蠕虫状EG表面, 压制成型过程中 $\mathrm{SA}$ 晶体发 生区域性堆积现象，均匀性较差.

\subsection{DSC分析}

图 4为纯 SA 和膨胀石墨 b配置的 EG含量 $15 \%$ 和 $20 \%$ 的样品 (密度 $900 \mathrm{~kg} \mathrm{~m}^{-3}$ ) 在熔化与凝固过程的 DSC曲线. 从图4(a)熔化曲线中可以看出, EG/SA复 合材料相对纯 $\mathrm{SA}$ 相转变温度 $\left(T_{\mathrm{o}}^{\mathrm{m}}\right)$ 略有增加, 这是由 于石墨微观结构的存在使得复合相变材料中 SA发生 相变时微单元结构内的压力增加导致 ${ }^{[12]}$; 相比EG含 量 $15 \%$ 的样品, EG含量 $20 \%$ 时相转变温度 $\left(T_{\mathrm{o}}^{\mathrm{m}}\right)$ 略有降 低, 这是因为更多的EG使 SA更加分散, 晶粒更小易 于熔化. 从图4(b)凝固曲线中可以看出, EG/SA复合 材料相对纯 $\mathrm{SA}$ 相转变温度 $\left(T_{\mathrm{o}}^{\mathrm{f}}\right)$ 略有增加, 同样为材 料内部压力增大所致; 相比EG含量 $15 \%$ 的样品, EG 含量 $20 \%$ 时相转变温度 $\left(T_{\mathrm{o}}^{\mathrm{f}}\right)$ 同样略有降低, 这是因为 EG含量变高, 石墨片层更加规则使得 $\mathrm{SA}$ 分子链段的 扩散受到更大的阻碍，不利于结晶.

对比图4(a)和(b)可以发现, 纯SA过冷度为 $1.86^{\circ} \mathrm{C}$, 复合储能材料过冷度为 $1.59 \sim 1.73^{\circ} \mathrm{C}$. 可见EG的异相 成核和传热强化作用可以促进SA发生相变时降低其 过冷度, 且EG含量越高, 促进作用越明显, 过冷度 越小. 通过对比纯 SA和复合材料的熔化焓以及凝固 焓, 可以发现SA的重结晶性能非常好, 凝固焓和熔 化焓相差很小, 且 EG的加人和压块处理并不会影响
SA本身的相变潜热，复合材料的相变焓与纯 SA相变 焓的比值近似等于其中 SA的质量分数, 例如: EG含 量为 $20 \%$ 的样品相变潜热为 $165.88 \mathrm{~J} \mathrm{~g}^{-1}$, 纯 $\mathrm{SA}$ 相变 潜热约为 $206.03 \mathrm{~J} \mathrm{~g}^{-1}$, 二者的比值约为 0.8 .

\section{3 热导率分析}

EG/SA定形相变储能复合材料的径向热导率如 图5所示，可以看出当EG含量为 $15 \%$ 时，两种EG配置 的样品在径向导热随密度的变化规律上有显著的差 别, a组样品在密度大于 $850 \mathrm{~kg} \mathrm{~m}^{-3}$ 后热导率开始下 降，这是由于密度过大使得压制过程中EG孔隙中部 分SA挤出包裹在EG片层周围，阻碍导热的作用开始 大于密度变大使得石墨片层规则增强径向导热的作 用; 而 $\mathrm{b}$ 组样品径向热导率则始终随密度的增加而增 大，这是因为 $\mathrm{EG}$ 膨胀度的增大提供了更大的孔体积， SA更为均匀和充分地吸附在EG的孔隙当中，压缩过 程中SA挤出相对较少且较为分散, 此时密度变大使 得石墨片层规则增强径向导热的作用占了主导地位. 当EG含量为 $20 \%$ 时, $a, b$ 两组样品径向热导率均随密 度的增大而增大, 且 $b$ 组样品热导率略大于同密度下 $a$ 组样品热导率, 在密度为 $950 \mathrm{~kg} \mathrm{~m}^{-3}$ 时, $b$ 组样品径 向热导率高达 $19.6 \mathrm{~W} \mathrm{~m}^{-1} \mathrm{~K}^{-1}$.

膨胀石墨的膨胀度对相变材料的稳定吸附倍率 具有重要影响, $600^{\circ} \mathrm{C}$ 膨胀制得的EG对石蜡的稳定吸 附倍率在5倍左右, 而 $900 \sim 1000{ }^{\circ} \mathrm{C}$ 膨胀制得的 $\mathrm{EG}$ 对 石蜡的稳定吸附倍率在 15 20倍 ${ }^{[13]}$. EG微孔对石蜡和 硬脂酸等有机物的吸附性能有一定的相似性, 当EG 质量分数为 $15 \%$ 时 (即 $\mathrm{EG:SA}$ 质量比为 $15: 85$ ), $600^{\circ} \mathrm{C}$ 膨胀制得的 $E G$ 微孔无法充分吸附所有 $S A$. 由于膨胀
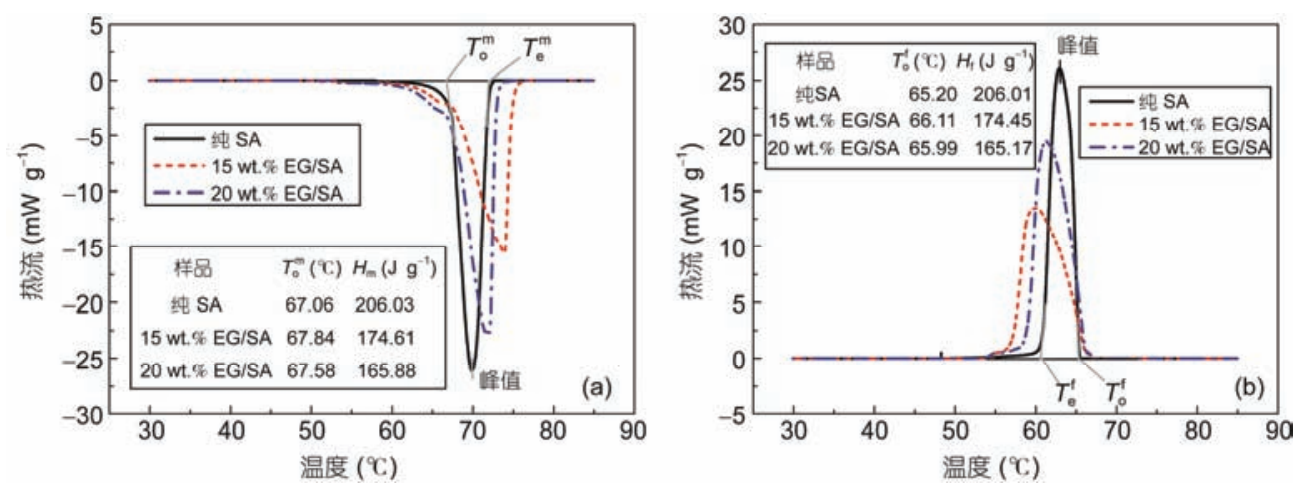

图 4 (网络版彩色)EG/SA定形相变储能复合材料熔化与凝固过程DSC曲线. (a) 熔化过程; (b) 凝固过程

Figure 4 (Color online) The DSC curve of form-stable EG/SA composite samples during melting and freezing processes. (a) Melting process; (b) freezing process 


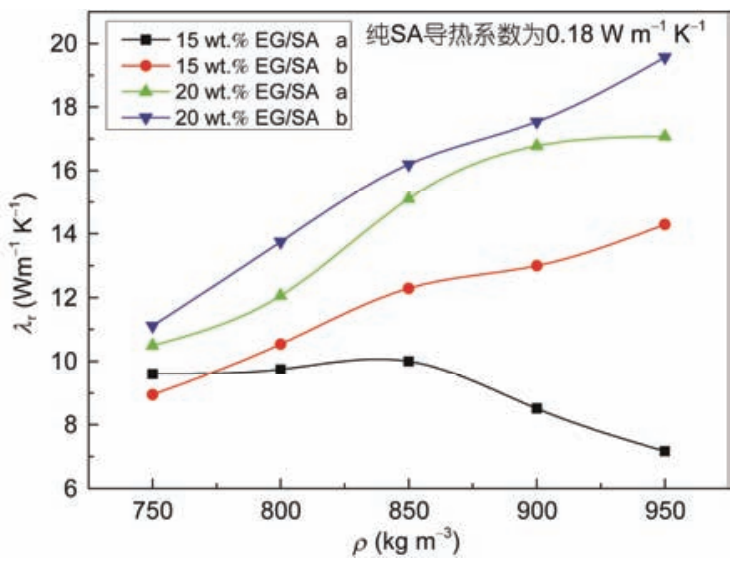

图 5 (网络版彩色) EG/SA定形相变储能复合材料径向导热系数随压 块密度变化曲线

Figure 5 (Color online) The radial thermal conductivities of formstable EG/SA composite samples at different packed densities

石墨孔体积随压块密度的增大逐渐减小, 当密度增 大致使膨胀石墨的孔体积小到无法容纳相变材料 SA 时, EG孔隙中的SA将被挤出影响导热系数的进一步 提高. 对于采用膨胀石墨 $\mathrm{a}$ 为多孔基体的相变储能复 合材料, 由于受膨胀度的限制当压块密度高于 $850 \mathrm{~kg}$ $\mathrm{m}^{-3}$ 时其导热系数随压块密度的增加反而减少; 而对 于采用膨胀石墨 $\mathrm{b}$ 为多孔基体的相变储能复合材料, 由于其丰富的微孔结构, 导热系数随压块密度增加 进一步增加, 可见EG膨胀度的增大对材料 径向热 导率的改善作用只有在密度较高时才有明显体现.

表1列出了近年来国内外研究中以EG为多孔基 体的相变储能复合材料的导热系数对比 (其中PA为 软脂酸 $)^{[11,12,15 ~ 21]}$. 可以看到 “熔融吸附-模压成型”的
方法相比其他制备方法在提高复合材料热导率上有着 非常大的优势, 采用高膨胀度EG制备的样品径向导 热系数有了进一步提高, 在EG质量分数 $20 \%$, 密度 $950 \mathrm{~kg} \mathrm{~m}^{-3}$ 时径向导热系数相比纯 $\mathrm{SA}\left(0.18 \mathrm{~W} \mathrm{~m}^{-1} \mathrm{~K}^{-1}\right)$ 提高了110倍.

\section{4 热稳定性分析}

相变储能复合材料的热稳定性对其储能性能有 重要影响, 储/放热循环实验发现, 在密度较小 $(750$, 800 和 $850 \mathrm{~kg} \mathrm{~m}^{-3}$ ) 时所有样品中SA均未发生明显的泄 漏现象, 即质量基本保持不变. 当成型密度较高时部 分样品会出现SA泄露现象, 图6为密度 900 和 $950 \mathrm{~kg}$ $\mathrm{m}^{-3}$ 的 EG/SA定形相变储能复合材料的质量随储/放 热循环次数的变化曲线.

通过图6(a)可以看到, 在EG含量 $15 \%$ 、密度为 900 和 $950 \mathrm{~kg} \mathrm{~m}^{-3}$ 时, $a$ 组样品均存在泄漏现象, 在循环 5 次后样品不再出现明显泄漏, SA泄漏质量占复合材 料总质量的 $10 \%$ 左右; $\mathrm{b}$ 组样品均未出现明显泄漏, 这也说明更高膨胀度的 $\mathrm{EG}$ 为 $\mathrm{SA}$ 提供了足够的孔隙, 压制成型后SA均匀分布在石墨片层中，熔化后不会 泄漏流出, 因此对于定形相变复合材料的循环热性 能有着很好的改善作用. 通过图6(b)可以看到, 在EG 含量 $20 \%$ 时, 所有样品均未发生明显的泄漏问题, 这 是因为此时膨胀石墨 $\mathrm{a}$ 为 $\mathrm{SA}$ 提供的孔体积较大, $\mathrm{SA}$ 相 变时有足够的空间容纳不会泄漏.

图7为储/放热循环实验中样品出现的几种形貌 变化. 从图中可以看到, 储/放热循环过程中样品出 现 4 种形态: 无变化、泄漏、开裂和裂为两半. 实验过

表 1 膨胀石墨基复合相变储能复合材料制备方法和导热系数对比

Table 1 The comparison of thermal conductivities of EG-based composite PCMs using different preparation methods

\begin{tabular}{|c|c|c|c|c|}
\hline 复合相变材料 & 制备方法 & 密度 $\left(\mathrm{kg} \mathrm{m}^{-3}\right)$ & 导热系数 $\left(\mathrm{W} \mathrm{m}^{-1} \mathrm{~K}^{-1}\right)$ & 参考文献 \\
\hline 8 wt. $\%$ EG/SA & 真空熔融吸附 & - & 2.52 & {$[11]$} \\
\hline 7.14 wt. $\%$ EG/SA-PA & 熔融吸附搅拌 & - & 2.51 & {$[15]$} \\
\hline 8 wt. $\%$ EG/SA-PA & 熔融混合电磁搅拌 & - & 4.43 & {$[16]$} \\
\hline 5 wt. $\%$ EG/SA & EG加入液态SA混合 & - & 3.2 & [17] \\
\hline $10 \mathrm{wt} . \% \mathrm{EG} / \mathrm{SA}$ & $\mathrm{EG}$ 加人液态 $\mathrm{SA}$ 振动摇匀 & - & 1.1 & {$[18]$} \\
\hline 20 wt. $\%$ EG/SA & 混合加热摚拌研细 & - & 2.9122 & [19] \\
\hline 20 wt. $\%$ EG/SA & EG加人液态 SA搅拌超声处理 & - & 1.577 & {$[20]$} \\
\hline 20 wt. $\%$ EG/PA & 真空浸渍 & - & 0.6 & {$[21]$} \\
\hline $20 \mathrm{wt} . \% \mathrm{EG} / \mathrm{SA}$ & 熔融吸附-模压成型 & 950 & 18.6 & {$[12]$} \\
\hline $20 \mathrm{wt} . \% \mathrm{EG} / \mathrm{SA}$ & 熔融吸附-模压成型 & 950 & 19.6 & 本研究 \\
\hline
\end{tabular}



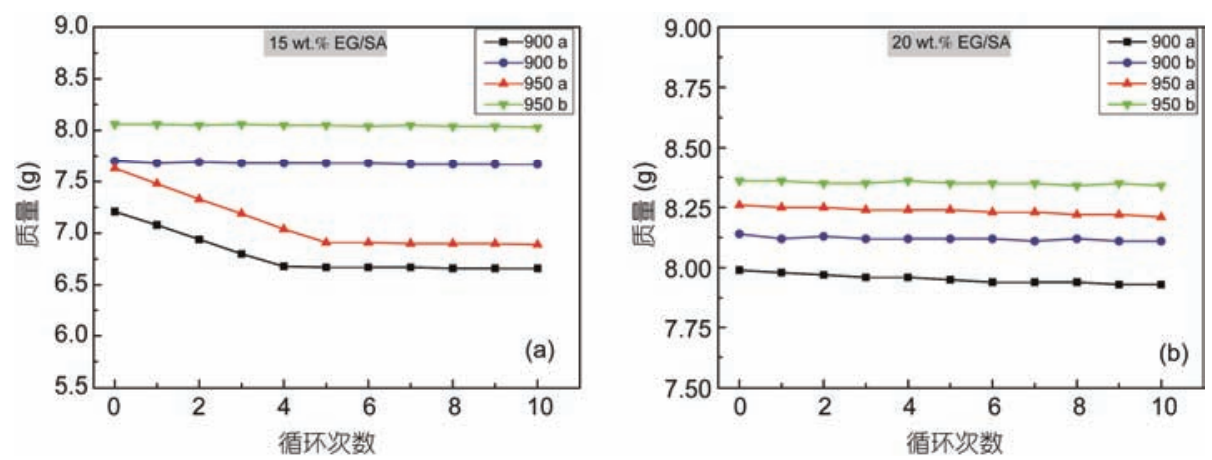

图 6 (网络版彩色)EG/SA定形相变储能复合材料的质量随储/放热循环次数的变化曲线. (a) 15 wt.\%EG/SA sample; (b) 20 wt.\% EG/SA sample Figure 6 (Color online) The mass variation of form-stable EG/SA composite samples at different cycle times. (a) 15 wt.\%EG/SA sample; (b) 20 wt.\% EG/SA sample

程中发现在循环5次左右后样品形态基本不再发生变 化, 大多数样品均出现不同程度的开裂, 尤其在密度 较高时开裂比较严重甚至裂为两半.

实验中观察发现, EG含量 $15 \%$ 时不出现明显开 裂的极限密度约为800 850 kg m${ }^{-3}$, EG含量 $20 \%$ 时不 出现明显开裂的极限密度约为850 900 $\mathrm{kg} \mathrm{m}^{-3}$. 这是 因为 SA熔化后体积有所增大, 未发生泄漏时会对石 墨片层产生一定膨胀压力, 使样品产生不同程度的 开裂, 因此, 提高 $\mathrm{EG}$ 的膨胀度和控制适宜的成型密 度是解决定形相变储能复合材料开裂问题的关键.

\section{5 储能单元装置储/放热性能分析}

图 8 为 $\mathrm{EG} / \mathrm{SA}$ 定形相变储能复合材料储/放热过 程单元装置温度变化曲线, 可以看出储能单元装置 从 $40{ }^{\circ} \mathrm{C}$ 升温至 $75^{\circ} \mathrm{C}$ 过程中(图 8(a)), EG/SA定形复合 材料的储热速率相比纯SA大幅度提高, 其储热时间 缩短为纯 SA的 1/6 1/4, 4种EG/SA定形复合材料均有 1 个斜率明显减小的相变区间平坦段, 其相变区间大 概在67.5 $69^{\circ} \mathrm{C}$. 而纯 SA曲线相变区间却不是特别明
显, 只是在 $65 \sim 69^{\circ} \mathrm{C}$ 斜率稍有所下降, 这是因为中心 点测温存在一定的滞后性, 即该处材料升温慢于周 围材料, 相比复合材料纯SA的热导率要低很多, 使 得这一点在其加热过程中体现非常突出. 从 $70 \sim 75^{\circ} \mathrm{C}$ 温升段曲线看可以发现虽然传热温差减小, 但复合 材料仍有比较高的斜率, 即升温较快, 说明其导热系 数并未因SA的熔化而明显减小; 同时纯SA在此温度 区间曲线也有着较大斜率，是因为 SA熔化后自然对 流作用缩短了其加热时间.

图 8(b)为 5 个单元装置从 $75^{\circ} \mathrm{C}$ 降温至 $40^{\circ} \mathrm{C}$ 过程中 装置内材料中心点温度变化曲线. 可以看到, EG/SA 定形复合材料的冷却速率相比纯 SA也变快了很多, 其放热时间缩短为纯SA的 $1 / 8 \sim 1 / 5$. 4种复合材料的放 热过程平坦段温度区间大概为 $67.5 \sim 68.5^{\circ} \mathrm{C}$, 相比储 热过程略低, 而过冷度小于DSC曲线测试结果, 这是 因为DSC中温降速率较大, 存在一定的滞后性. 纯 $\mathrm{SA}$ 在初始阶段降温速率很快, 与 $\mathrm{EG} / \mathrm{SA}$ 复合材料不 同，纯 SA在相变温度区间段没有出现平坦区间，且 整体温降耗时仍然很长. 相关差异主要是因为EG/SA
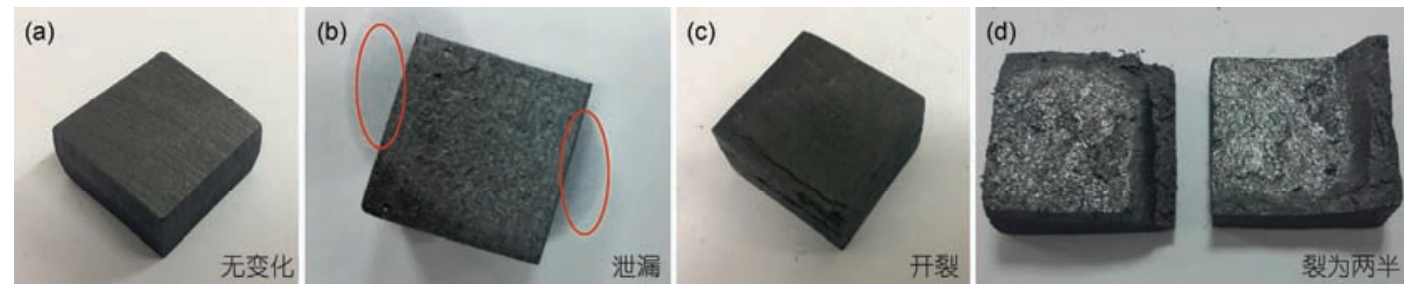

图 7 (网络版彩色)EG/SA定形相变储能复合材料储/放热循环中相貌变化. (a) $20 \mathrm{wt} . \% \mathrm{EGb} / \mathrm{SA}$ 样品 $750 \mathrm{~kg} \mathrm{~m}{ }^{-3} ;$ (b) $15 \mathrm{wt}$. $\% \mathrm{EGa} / \mathrm{SA}$ 样品 $900 \mathrm{~kg}$ $\mathrm{m}^{-3}$; (c) $15 \mathrm{wt} . \% \mathrm{EGb} / \mathrm{SA}$ 样品 $900 \mathrm{~kg} \mathrm{~m}^{-3}$; (d) $20 \mathrm{wt} \% \mathrm{EGa} / \mathrm{SA}$ 样品 $950 \mathrm{~kg} \mathrm{~m}^{-3}$

Figure 7 (Color online) The change of appearance form-stable EG/SA composite samples in the process of heat storing/releasing cycle. (a) 20 wt. $\% \mathrm{EGb} / \mathrm{SA}$ sample with $750 \mathrm{~kg} \mathrm{~m}^{-3}$; (b) $15 \mathrm{wt} . \% \mathrm{EGa} / \mathrm{SA}$ sample with $900 \mathrm{~kg} \mathrm{~m}^{-3}$; (c) $15 \mathrm{wt} \% \mathrm{EGb} / \mathrm{SA}$ sample with $900 \mathrm{~kg} \mathrm{~m}{ }^{-3}$; (d) 20 wt.\%EGa/SA sample with $950 \mathrm{~kg} \mathrm{~m}^{-3}$ 

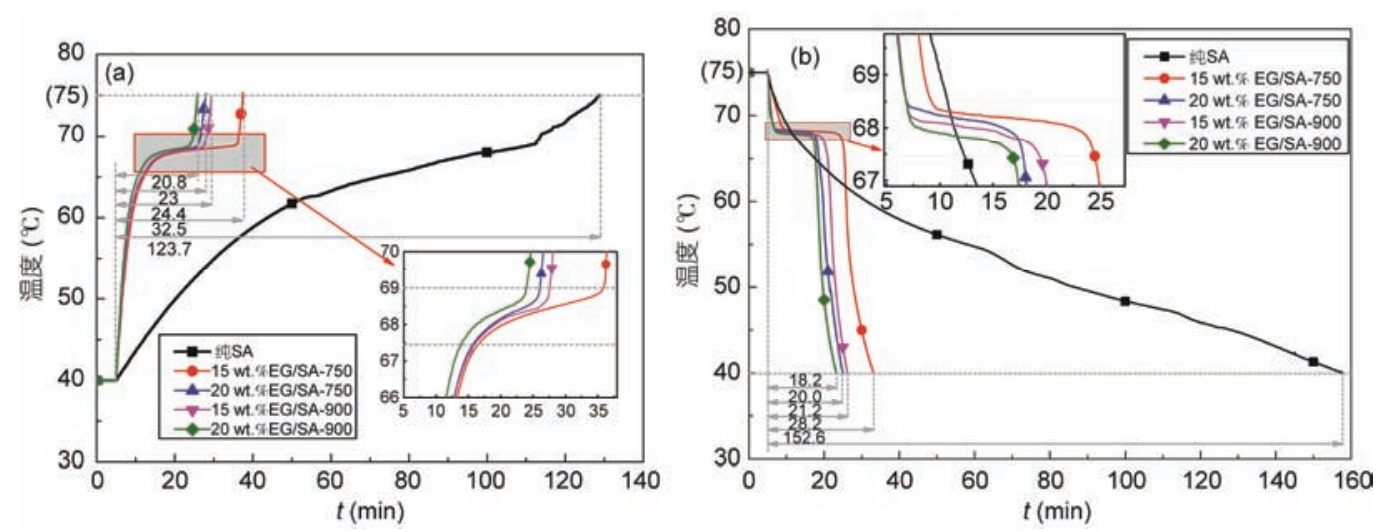

图 8 (网络版彩色)EG/SA定形相变储能复合材料储/放热过程单元装置温度变化曲线. (a) 储热过程; (b) 放热过程

Figure 8 (Color online) Temperature variations of SA and form-stable EG/SA composite samples versus time in charging/ discharging phase. (a) Charging phase; (b) discharging phase

复合材料具有很好的导热性能, 当温度降低到SA的 相变温度时, 复合材料中的大部分SA同时发生相变 释放大量热量, 当外界冷却功率低于相变材料的放 热功率时无法及时带走热量, EG/SA复合材料的温度 在相变区间保持平稳; 当相变过程快结束时, 冷却功 率高于相变材料的放热功率因此温度又重新快速下 降. 而对于纯相变材料 SA, 在初始阶段没有发生相 变前, 由于传热温差较大液态 SA的自然对流换热作 用较强使得其降温速率很快, 随着温度进一步降低, 靠近换热壁面的相变材料 SA最先冷却到相变温度释 放热量, 由于此时纯SA的自然对流相对前期已弱化 以及 $\mathrm{SA}$ 导热系数很低, 远离换热壁面的相变材料 SA 温度较高没有发生相变释放热量, 因此纯SA的相变 过程从换热壁面到中心位置逐渐进行, 因此瞬时释放 的热量较低, 外界冷却功率仍高于相变材料的放热功 率，因而在相变温度区间段没有出现平坦区间.

表 2 列出了整个储/放热循环中 5 个储能单元装置 的性能参数, 可以发现 4 种 EG/SA复合材料放热时间 相比储热时间略有缩短, 即平均放热功率要大于平
均加热功率，这是因为 $\mathrm{EG}$ 的异相成核能加快复合材 料的结晶速率, 而其物理吸附作用会使 SA熔化活化 能有所升高不利于熔化 ${ }^{[11]}$. 纯SA放热时间相比储热 时间却有明显增长, 这是因为储热过程中自然对流 作用发生在小温差区间大大减少了 $69 \sim 75^{\circ} \mathrm{C}$ 温度段的 升温时间; 而冷却过程中自然对流作用发生在大温差 区间，此时本身换热就较快，温差较小时SA已完全凝 固，此时换热非常缓慢，大大增加了放热时间.

同时可以看出, 填充 $15 \mathrm{wt} \%$ EG/SA-900 $\mathrm{kg} \mathrm{m}^{-3}$ 复合材料的装置升降温时间长于 $20 \mathrm{wt} \% \mathrm{EG} / \mathrm{SA}-750$ $\mathrm{kg} \mathrm{m}^{-3}$ 复合材料的装置, 而径向热导率测试结果显示 导热系数前者比后者大, 这是因为实验中为控制传热 面积相同, 单元装置中复合材料体积相同, 故而 5 个单 元装置中SA质量不同, 而15 wt.\%EG/SA-900 $\mathrm{kg} \mathrm{m}^{-3}$ 装 置中SA含量较多, 总储热量大, 升降温相对较慢.

从上表可以看出储能单元装置平均储/放热功率 和装置内材料导热系数呈正相关, 纯SA由于熔化后 流动产生对流作用使其平均储/放热功率有所增加, 其中 $20 \mathrm{wt}$.\%EGb/SA-900 $\mathrm{kg} \mathrm{m}^{-3}$ 单元装置平均储热功

\section{表 2 EG/SA定形相变储能复合材料储能单元装置储/放热循环性能参数}

Table 2 Performance of form-stable EG/SA composite samples in energy storage unit during the charging and discharging processes

\begin{tabular}{lcccccc}
\hline 单元装置 $\left(\mathrm{kg} \mathrm{m}^{-3}\right)$ & 总储热量 $(\mathrm{kJ})$ & 储热时间 $(\mathrm{min})$ & 平均储热功率 $(\mathrm{W})$ & 放热时间 $(\mathrm{min})$ & 平均放热功率 $(\mathrm{W})$ & 导热系数 $\left(\mathrm{W} \mathrm{m}^{-1} \mathrm{~K}^{-1}\right)$ \\
\hline 纯SA(847) & 118.38 & 123.7 & 15.95 & 152.6 & 12.93 & 0.18 \\
15 wt.\%EGb/SA-750 & 90.50 & 32.5 & 46.41 & 28.2 & 53.49 & 8.951 \\
20 wt.\%EGb/SA-750 & 85.71 & 23.0 & 62.11 & 20.0 & 71.43 & 11.116 \\
15 wt.\%EGb/SA-900 & 108.58 & 24.4 & 74.17 & 21.2 & 85.36 & 12.998 \\
20 wt.\%EGb/SA-900 & 102.86 & 20.8 & 82.42 & 18.2 & 94.19 & 17.536 \\
\hline
\end{tabular}


率为纯 $\mathrm{SA}$ 单元装置的 5.5 倍, 平均放热功率为纯 $\mathrm{SA}$ 单 元装置的7.3倍, 换热效果增强显著.

\section{3 结论}

本文研究了两种不同膨胀度膨胀石墨对膨胀石 墨/硬脂酸定形相变储能复合材料的热性能影响规律, 采用“熔融吸附-模压成型”方法制备膨胀石墨基定形 相变储能复合材料样品, 通过对不同参数样品微观形 貌、相变温度/潜热、径向导热系数和热稳定性的表征 和测试, 探究了高膨胀度膨胀石墨对定形相变复合材 料热性能的影响与改善作用. 最后, 通过纯硬脂酸及 复合材料单元装置的水浴储/放热实验测试了定形相 变储能复合材料的实际换热性能. 主要得到以下结论.

(1) 微观形貌显示膨胀石墨膨胀度的增大使得 复合相变材料中硬脂酸的吸附更加充分, 压制后能 够更加均匀地附着在石墨片层中. DSC测试结果显示 $\mathrm{EG}$ 的加人和压块处理使 $\mathrm{SA}$ 相变温度略有增加, 对于 SA本身的相变潜热影响较小, 同时EG的异相成核和
传热强化作用可以促进SA发生相变, 降低其过冷度.

(2) 膨胀石墨膨胀度的增大对定形相变储能复 合材料的径向热导率起到了很大的改善作用，在EG 含量 $15 \%$ 时样品径向热导率不再随密度的增加而下 降, 这对于兼顾高的体积储热密度和热导率有着非 常重要的意义; 高膨胀度EG制得的 20 wt.\%EGb/SA$950 \mathrm{~kg} \mathrm{~m}^{-3}$ 定形相变储能复合材料, 其径向导热高达 $19.6 \mathrm{~W} \mathrm{~m}^{-1} \mathrm{~K}^{-1}$, 相比纯SA提高了 110 倍.

(3) 膨胀石墨膨胀度的增大对定形相变储能复 合材料的循环热稳定性也起到了很大的改善作用, 在EG含量为 $15 \%$ 时, 高密度样品(密度 $\geqslant 900 \mathrm{~kg} \mathrm{~m}^{-3}$ ) 不再发生 SA的泄漏，定形封装效果良好; 定形相变 储能复合材料相比纯硬脂酸换热效果增强显著, 其 中 $20 \mathrm{wt} . \% \mathrm{EGb} / \mathrm{SA}-900 \mathrm{~kg} \mathrm{~m}^{-3}$ 单元装置平均储热功率 为纯 SA单元装置的 5.5 倍, 平均放热功率为纯 SA单元 装置的 7.3 倍. 定形相变储能复合材料的升降温时间 大大缩短, 约为纯相变材料的 $1 / 8 \sim 1 / 4$, 具有高导热、 无泄漏等优点.

\section{参考文献}

1 Li T X, Lee J H, Wang R Z, et al. Enhancement of heat transfer for thermal energy storage application using stearic acid nanocomposite with multi-walled carbon nanotubes. Energy, 2013, 55: 752-761

2 Liu M, Saman W, Bruno F. Validation of a mathematical model for encapsulated phase change material flat slabs for cooling applications. Appl Therm Eng, 2011, 31: 2340-2347

3 Wang X, Zhang Y P, Xiao W, et al. Review on thermal performance of phase change energy storage building envelope. Chin Sci Bull, 2009, 54: 920-928

4 Shukla A, Buddhi D, Sawhney R L. Solar water heaters with phase change material thermal energy storage medium: A review. Renew Sust Energ Rev, 2009, 13: 2119-2125

5 Shaikh S, Lafdi K. C/C composite, carbon nanotube and paraffin wax hybrid systems for the thermal control of pulsed power in electrics. Carbon, 2010, 48: 813-824

6 Zhang Y, Zhang X S. The development of the study of energy storage using oganic phase change materials (in Chinese). Acta Energ Sin, 2006, 27: 725-730 [张奕, 张小松. 有机相变材料储能的研究和进展. 太阳能学报, 2006, 27: 725-730]

7 Zhao C Y, Pan Z H, Wang Q, et al. Heat transfer of phase change materials (PCMs) and thermochemical heat storage in porous materials (in Chinese). Chin Sci Bull, 2016, 61: 1897-1911 [赵长颖, 潘智豪, 王倩, 等. 多孔介质的相变和热化学储热性能. 科学通报, 2016, 61: 1897-1911]

8 Wang S P, Xu T, Gao X N, et al. Recent progress about expanded graphite matrix composite phase change material for energy storage (in Chinese). Energy Stor Sci Technol, 2014, 3: 210-215 [王淑萍, 徐涛，高学农，等. 膨胀石墨基复合相变储能材料的研究进展. 储能 科学与技术, 2014, 3: 210-215]

9 Gao X N, Li D L, Sun T, et al. Performance of temperature-controlled electronic heat sink with composite paraffin/expanded graphite phase change material (in Chinese). J South China Univ Technol: Nat Sci Ed, 2012, 40: 7-12 [高学农, 李得伦, 孙滔, 等. 石蜡/膨胀石 墨复合相变材料控温电子散热器的性能. 华南理工大学学报(自然科学版), 2012, 40: 7-12]

$10 \mathrm{Hu}$ P, Yu J J, Wang X W. Preparation and thermal performance of palmitic acid/expanded graphite composite phase change materials (in Chinese). J Eng Thermophys, 2017, 38: 464-469 [胡芒, 于晶晶, 王向往. 棕榈酸/膨胀石墨复合相变材料的制备及性能研究. 工程 热物理学报, 2017, 38: 464-469]

11 Zhou W B, Zhang L, Zhu J Q, et al. Kinetics study of phase change stearic acid/expanded graphite composite as heat storage material (in 
Chinese). J Wuhan Univ Technol, 2012, 34: 9-13 [周卫兵, 张否, 朱教群, 等. 硬脂酸/膨胀石墨复合相变储热的动力学研究. 武汉 理工大学学报, 2012, 34: 9-13]

12 Wu S, Li T X, Yan T, et al. Preparation and thermal properties of high performance shape-stabilized phase change composites using stearic acid and expanded graphite (in Chinese). CIESC J, 2015, 66: 5127-5134 [仵斯, 李廷贤, 闰霆, 等. 高性能定形复合相变储能 材料的制备及热性能研究. 化工学报, 2015, 66: 5127-5134]

13 Sun K, Yan F M, Zhang B N, et al. Preparation and study on the expanded graphite/paraffin composite phase change thermal energy storage material (in Chinese). New Chem Mater, 2012, 40: 148-150 [孙凯, 晏凤梅, 张步宁, 等. 膨胀石墨/石蜡复合相变储能材料的 制备与性能研究. 化工新形材料, 2012, 40: 148-150]

14 Wang H N, Zheng Y P, Kang F Y. Quantitative analysis of the pore structure of expanded graphite (in Chinese). J Inorg Mater, 2003, 18: 606-612 [王海宁, 郑永平, 康飞宇. 膨胀石墨孔结构的定量研究. 无机材料学报, 2003, 18: 606-612]

15 Zhang N, Yuan Y P, Du Y X, et al. Preparation and properties of palmitic-stearic acid eutectic mixture/expanded graphite composite as phase change material for energy storage. Energy, 2014, 78: 950-956

16 Yuan Y P, Zhang N, Li T Y, et al. Thermal performance enhancement of palmitic-stearic acid by adding graphene nanoplatelets and expanded graphite for thermal energy storage: A comparative study. Energy, 2016, 97: 488-497

17 Li T X, Lee J H, Wang R Z, et al. Heat transfer characteristics of phase change nanocomposite materials for thermal energy storage application. Int J Heat Mass Tran, 2014, 75: 1-11

18 Karaipekli A, Sari A, Kaygusuz K. Thermal conductivity improvement of stearic acid using expanded graphite and carbon fiber for energy storage applications. Renew Energ, 2007, 32: 2201-2210

19 Yuan P, Fu L, Tian Z, et al . Preparation and storage behavior of stearic phase change material (in Chinese). Guangzhou Chem Ind, 2015, 43: 105-107 [袁萍, 傅蕾, 田哲, 等. 硬脂酸定型相变材料的制备与储能行为的研究. 广州化工, 2015, 43: 105-107]

20 Choi B K, Ko J K, Park S J, et al. Influences of expanded graphite on thermal characterizations of erythritol, paraffin, and stearic acid for low-temperature phase change material composites. Int J Eng Sci Res Technol, 2016, 5: 796-807

21 Sari A, Karaipekli A. Preparation, thermal properties and thermal reliability of palmitic acid/expanded graphite composite as form-stable PCM for thermal energy storage. Sol Energ Mat Sol C, 2009, 93: 571-576 


\title{
Preparation and thermal performance of form-stable expanded graphite/stearic acid composite phase change materials with high thermal conductivity
}

\author{
Tianyao Zhai, Tingxian $\mathrm{Li}^{*}, \mathrm{Si}$ Wu \& Ruzhu Wang \\ Institute of Refrigeration and Cryogenics, Shanghai Jiao Tong University, Shanghai 200240, China \\ * Corresponding author, E-mail: Litx@sjtu.edu.cn
}

Thermal energy storage plays an important role in improving the working reliability and utilization efficiency of renewable energy systems. Thermal energy storage includes sensible heat storage, latent heat storage using phase change material (PCM) and thermochemical heat storage. In comparison with sensible heat storage, latent heat storage has the distinct advantages of its high energy storage density and stable temperature during the energy storage and release processes. However, low thermal conductivity of PCM is the common drawback for various latent heat energy storage systems. The purpose of the research is to enhance the thermal conductivity of PCM by developing new form-stable composite phase change materials using expanded graphite matrix. Firstly, a preparation method "heating adsorption of liquid SA into expanded graphite (EG) matrix and compressing stable-shape composite" is employed to synthesis formstable composite phase change material. Twenty samples with different EG contents and packed densities are prepared using stearic acid (SA) as phase change material and two kinds of EG with different dilatation as porous matrix. Secondly, the microstructures, thermal properties and thermal stabilities of different EG/SA samples are tested to analyze the influence of the dilatation of expanded graphite on the thermal performance of form-stable composite phase change materials. Moreover, EG/SA form-stable heat storage units with different EG contents and packed densities are fabricated to analyze the thermal storage/release performance through heating/cooling experiment. The microstructures of EG and EG/SA samples are observed using scanning electron microscopy (SEM) and the results show there exist obvious differences between two kinds of EG and SA distribution in the samples. The EG/SA samples with high dilatation EG are even more uniform because the high dilatation EG has larger pore size and pore volume. The data obtained from differential scanning calorimetry (DSC) show the addition of EG and compressing operation have negligible effect on the latent heat and slightly increase the phase transition temperature of pure SA. The form-stable EG/SA composite PCMs have lower supercooling degree than pure SA. LFA447 flash thermal diffusivity instrument is used to evaluate the effect of EG's dilatation on the thermal conductivity of EG/SA samples. The results show that the use of high dilatation EG is more effective to improve the thermal conductivity of form-stable EG/SA composite PCMs when packed density is large. The radial thermal conductivity of form-stable $20 \mathrm{wt}$. EG/SA phase change composite with packed density of $950 \mathrm{~kg} \mathrm{~m}^{-3}$ is as high as $19.6 \mathrm{~W} \mathrm{~m}^{-1} \mathrm{~K}^{-1}$ when high dilatation EG is utilized. This figure is more than 110 times higher than that of pure SA. Finally, the thermal stability of EG/SA samples is conducted by repeating the charging and discharging processes of SA. These samples prepared by high dilatation EG have better thermal stability than the samples prepared by low dilatation EG. The EG with high dilatation can assure the uniform distribution of SA inside EG/SA sample and thus prevent the leakage of liquid SA during the phase change transformation process due to its dilated microstructure. The heating/cooling test results show that the heat storage/release time of EG/SA composite units is about 1/8-1/4 of that of pure SA unit, and this means the heat transfer can be significantly enhanced. It suggests that the form-stable EG/SA composite phase change materials prepared by high dilatation EG has good comprehensive performance due to its high thermal conductivity, good thermal stability and no leakage problem.

expanded graphite, stearic acid, phase change energy storage, form-stable composite, thermal conductivity, thermal performance

doi: 10.1360/N972017-00831 